Ann. Zuotech., I976, 25 (2), 28I-286.

NOTE

\title{
INFLUENCE DE LA DISTRIBUTION \\ SOUS FORME SOLIDE OU LIQUIDE D'UN SUPPLÉMENT RICHE EN PROTÉINES CHEZ LE VEAU RUMINANT
}

\author{
R. GUII.HERMIE, P. PATLREAL-MIRAND* et R. TOILLEC \\ aver la collaboration technique de Marguerite Bratus \\ Station de Recherches rootechniques, \\ Cintri de Recherchos de Rennes, I. N.R.A., \\ 65 , rue de Saint-Bricuc, \\ 35042 Rennes Cedex \\ * Laboratoive d'Ëtudes du Métabolisme asoté, \\ Centre de Recherches de Clermont-Iirrand, I. N. R. A., \\ Theix, Saint Genès Champanelle, (j3111 Beaumont
}

\section{RÉSUMÚ}

Douze veaux mâles de race Frisonne ont été sevrés à l'âge de 6 semaines. I!s ont été répartis en 2 lots une semaine avant le sevrage. Ils ont alors reçu pendant une période expérimentale de I I semaines un aliment concentré ainsi qu'un supplément protéique (67 p. roo de tourteau de soja cuit, 32 p. roo de poudre de lactosérum et I p. Ioo de méthionine) distribué sous forme solide (lot "Supplément solide") ou sous forme liquide (lot "Supplément liquiden).

La distribution du supplément sous lorme liquide a entrainé une diminution importante (le l'urémie plasmatique (2 $\mathrm{I} \mathrm{mg} / \mathrm{r}$ oo $\mathrm{ml}$ au lieu de $38,2 \mathrm{~h}$ après le repas). Elle a également provoqué un accroissement des teneurs sanguines en la plupart des acides aminés indispensables (fig. I), particulièrement en méthionine $\left(3,3+\mathrm{mg}\right.$ de méthionine/ I oo $\mathrm{ml} \mathrm{au}$ lieu de $0,4^{0}, 4^{\mathrm{h}}$ après le repas). La forme liquide a donc permis de faire passer directement le supplément protéique dans la caillette ct d'éviter sá clégradation dans le rumen. Cependant, l'amélioration observéc dans la croissance (2.9 p. I oo) et l'efficacité alimentaire $(4,4$ p. I0o) n'a pas été significative (tabl. I).

\section{IN'TRODUC'TION}

Chez le ruminant, l'efficacité nutritionnelle des protéines alimentaires de bonne qualité peut être accrue en réduisant leur dégradation dans le rumen. Cela peut être obtenu soit en protégeant les protéines par tannage (I,EROY et ZEI,TER, I970) ou par 
traitement au formol (VÉRITÉ, Hoden et JOURNÉr, I974), soit encore en les distribuant sous forme liquide, de manière à les faire passer directement dans la caillette, grâce au rapprochement des lèvres de la gouttière oesophagienne (RAVEx et RoBINSON, I964; Orskov, Fraser et Corse, I970). Nous avons étudié chez le veau sevré précocement, l'influence du mode de distribution, solide ou liquide, d'un supplément protéique sur la croissance et 1'efficacité alimentaire, ainsi que sur les teneurs sanguines en urée et en acides aminés libres qui sont susceptibles de nous renseigner sur la digestion et l'utilisation métabolique des matières azotées alimentaires.

\section{MATÉRIEL ET MÉTHODES}

Douze veaux mâles de race Frisonne ont été achetés à l'âge d'environ une semaine ct maintenus en cases individuelles sur aire paillée pendant 15 semaines. Ils ont été sevrés à l'issue de la cinquième semaine de présence, après avoir consommé $27 \mathrm{~kg}$ de matière sèche d'aliment d'allaitement. Deux lots ("Supplément solide " et "Supplément liquide ") de 6 veaux ont été constitués une semaine avant le sevrage. Tous les animaux ont alors reçu pendant une période d'essai de I I semaines un aliment concentré riche en céréales $\left({ }^{1}\right)$ et un supplément protéique $\left({ }^{2}\right)$ distribués respectivement à raison de 3 parties du premier pour $\mathbf{I}$ du second (poids/poids). L'aliment concentré a été offert aux veaux des 2 lots, sous forme de granulés de $7 \mathrm{~mm}$ de ciamètre, une fois par jour, à $8 \mathrm{~h} 30 \mathrm{du}$ matin.

Le supplément protéique a été incorporé dans l'aliment concentré des veaux du lot "Supplément solide". Il a été dilué dans l'eau à raison de $200 \mathrm{~g}$ pour soo d'eau et distribué au seau une fois par jour à $8 \mathrm{~h}$ du matin, aux animaux du lot "Supplément liquide "; jusqu'au sevrage, il a été mélangé au lait de remplacement, afin d'habituer les animaux à le consomner. Les quantités d'aliment concentré et de supplément protéique offertes ont été très voisines de celles que peuvent ingérer des veaux mâles recevant un régime riche en aliment concentré (MATHIEv et WeGATLITRE, I96I) ; elles ont ainsi augmenté progressivement de 0,5 à 2,8 p. Ioo du poids vif, de la première à la dernière scmaine d'essai. En outre, I $50 \mathrm{~g}$ de foin ont été clistribués aux veaux des 2 lots à partir de la quatrième semaine d'essai.

Des prises de sang ont été effectuées dans la veine jugulaire, au cours de la huitième semaine l'essai, o, 2, 4 et 6 heures après la distribution des aliments. L'urée plasmatique a été dosée dans tous les échantillons et les acides aminés libres dans des échantillons moyens constitués par mélangc en quantités égales du sang prélevé sur les animaux d'un même lot au temps $+\mathrm{h} ;$ les méthodes de dosage utilisées ont été décrites par ailleurs (PATUREAU-Miraxi) at al., 1974).

\section{RÉSULTATS ET DISCUSSION}

L'état sanitaire des veaux a toujours été satisfaisant. Les animaux du lot « Supplément liquide" ont eu tendance à ingérer un peu moins d'aliment concentré (I,9 p. IOo) et à croître plus rapidement (2,9 p. IOO) avec une meilleure efficacité alimentaire (4,4 p. IOo) que les veaux du lot "Supplément solide " (tabl. I). Ces différences ont été maximales à l'issue de la huitième semaine d'essai : ainsi, l'écart observé dans le gain de poids vif cumulé a alors atteint $95 \mathrm{~g} / \mathrm{j}$ (I2,8 p. Ioo). Cependant, aucune de ces différences n'a été significative, contrairement à l'amélioration qui avait été

(1) Composition (p. roo) : orge: 35 , blé: 34 , luzerne : 27 et complément minćral vitaninisé : 4 ; teneur en matières azotées totales : I 2,8 p. Ioo/MS.

$\left({ }^{2}\right)$ Composition (p. Ioo) : tourteau de soja cuit : 67, poudre de lactosérum : 32, methimine commerciale: I ; teneur en matières azotées totales: $42 \mathrm{p}$. Ioo/MS. 
TABISAU I

Croissance, consommation et effcacité alimentaire au cours de la période d'essai

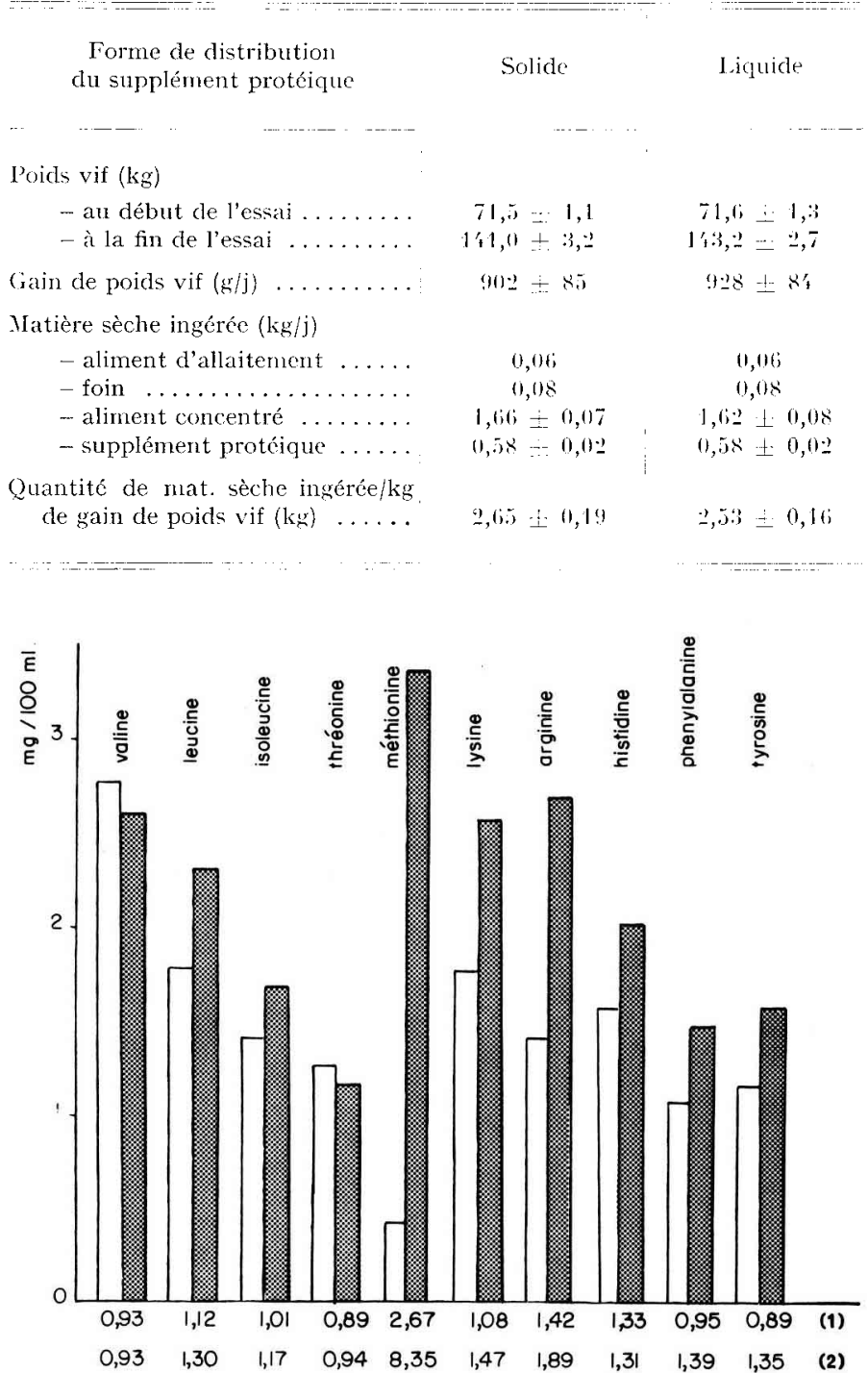

Fí.i. r. - Influence de la forme de présentation du supplément protéiqué sur la teneur du sang en acides aminés libres

(1) Teneur en acide aminé considéré des protéines du supplément/teneur des protéines du contenu de duodénum de bovin recevant un régime riche en aliment concentré (Champredon, Piox et THIVEND, I97I).

(2) Teneur en acide aniné considéré daus le sang des veaux du lot "Supplénont liquide "/teneur dans le sany des veaux du lot "Supplément solide".

Surface grisée: Lot "Supplénent liquide"

Surface claire: Lot "Supplénent solide" 
observée dans la croissance et l'efficacité alimentaire par Pryor et TERNouth (1972) en distribuant à des veaux un supplément riche en poudre de lait entier.

L'urémie a été plus élevée chez les animaux du lot "Supplément solide " (en moyenne $25,4 \pm \mathrm{I} 2,3 \mathrm{mg}$ p. IoO $\mathrm{ml}$ au lieu de $\mathrm{I} 6,7 \pm 7,4)(\mathrm{p} \leqslant 0,0 \mathrm{I})$. Cette différence a été particulièrement importante $2 \mathrm{~h}$ après $1 \mathrm{a}$ distribution des aliments, les valeurs obtenues atteignant alors respectivement $38,5 \pm \mathrm{I} 2$, I et $20,7 \pm 8,4 \mathrm{mg}$ p. Ioo $\mathrm{ml}$ $(p \leqslant 0,0 I)$. Cela traduit sans doute la désamination rapide des protéines du soja et du lactosérum dans le rumen des veaux du lot "Supplément solide ", compte tenu du taux protéique élevé de la ration.

Les teneurs sanguines en leucine, tyrosine, phénylalanine, lysine, histidine, arginine et surtout en méthionine ont été plus élevées chez les veaux du lot "Supplément liquide " que chez ceux du lot "Supplément solide " (fig. I). Les valeurs observées chez les veaux du lot "Supplément liquide " ont été très voisines de celles obtenues chez des veaux préruminants de même âge qui recevaient un lait de remplacement dont la majeure partie des protéines provenait du tourteau de soja supplémenté en méthionine (Patureau-Mirand, Prugnaud et Pion, I97I). Fin revanche, les concentrations en ces mêmes acides aminés dans le sang des veaux du lot "Supplément solide " ont été très proches de celles observées chez la vache laitière (CHAMPREDON, Pion et Journet, I970) ou chez le veau ruminant (Lisibholz et Nayror, I97I). Aucune différence importante n'a été mise en évidence entre les animaux des 2 lots en ce qui concerne les teneurs sanguines en thréonine, valine et isoleucine.

Les écarts observés dans les teneurs sanguines en acides aminés indispensables pourraient s'expliquer en partie, par les différences de composition en ces acides aminés entre les protéines du supplément et celles du contenu de duodénum des veaux du lot "Supplément solide ". La composition en acides aminés des matières azotées du contenu de duodénum de ces derniers peut-être estimée d'après les valeurs observées chez des bovins recevant une ration voisine, riche en aliments concentrés (Champredon, Pion et Thivend, i97I). Ainsi, lorsque la teneur en un acide aminé indispensable est plus élevée dans les protéines du supplément que dans celles du contenu de duodénum de ces bovins, la teneur sanguine en cet acide aminé est également plus élevée chez les veaux du lot "Supplément liquide " que chez ceux du lot "Supplément solide ", et réciproquement (fig. I).

Seuls les acides aminés aromatiques font exception. La distribution du supplément protéique a donc permis d'accroître les concentrations sanguines de certains acides aminés indispensables tels que la méthionine et la lysine qui pourraient être limitants chez les bovins en croissance, ainsi que celle en histidine, généralement peu élevée dans le sang des ruminants.

Ces résultats confirment le maintien du réflexe de rapprochement des lèvres de la gouttière œsophagienne chez le ruminant habitué à recevoir un aliment liquide même dépourvu de lait (@Rskov et BenziE, I969; Guir.hirmft, Mathiec et ToulLEC, I975). Ils confirment aussi la meilleure utilisation métabolique des protéines de bonne qualité lorsqu'elles sont digérées selon les mécanismes du monogastrique (RAven et Robinson, I964; Orskov, Fraser et Corse, r970).

Il est cependant possible que l'augmentation que nous avons observée dans les concentrations sanguines en acides aminés indispensables ne soit qu'éphémère et soit due à l'ingestion massive du supplément protéique en un seul repas. Ińn revanche, il se pourrait que l'augmentation réelle des quantités de protéines fixées soit plus 
inportante que ne l'indiquerait l'accroissement du gain de poids vif ; en effet, BLACK et TRIBE (I973) ont observé chez l'Agneau une diminution dans le poids du réticulorumen, en infusant une partie de la ration dans la caillette.

En conclusion, la distribution d'une part des protéines alimentaires sous forme liquide permet de réduire leur dégradation dans le rumen et d'améliorer leur utilisation métabolique. Des études complémentaires sont nécessaires pour préciser l'intérêt zootechnique et les modalités pratiques d'utilisation de ce procédé.

$$
\text { Ręu pour publication en décrmbre } 1975 .
$$

\section{SUMMARY}

\section{EFFECT OF FEEDING A RICH PROTEIN SUPPIEMEXT EITHER SOLID OR LIQUID IN RUMINANT CAI,VES}

Twelve male Friesian calves were weaned at the age of 6 weeks. They were distributed into 2 groups one week before weaning. Thereafter, for an experimental period of I I weeks, the animals received a concentrate feed as well as a protein supplement (67 p. Ioo cooked soyabean meal, 32 p. Ioo whey powder and I p. Ioo methionine) offered either solid (" solid supplement "group) or liquid (" liquid supplement " group).

Administration of the supplement in liquid form led to a large decrease in the plasma level of urea (2I mg/roo $\mathrm{ml}$ versus 38 , two hours after the meal). It also brought about an increase in the blood levels of most of the essential amino acids (fig. I), especially methionine $(3.34 \mathrm{mg}$ methionine/100 ml versus $0.4^{\circ}$, four hours after the meal). Thus, the protein supplement, when liquid, arrived directly in the abomasum and avoided therefore degradation within the rumen. However, the improvement observed with respect to growth $(2.9 \mathrm{p}$. Ioo) and feed efficiency $(+.+$ P. Ioo) was not significant (table $I$ ).

\section{RÉFÉRENCES BIBLIOGRAPHIQUES}

Heack J. L., Tribl: I). E., I973. Comparison of ruminal and abomasal adninistration of feed on the: growth and body composition of lambs. Austr. J. A gric. Res., 24, 763-773.

Champridon C., l'ION R., Journet M., i97o. Influence de la nature des matières azotées alimentaires sur l'aninoacidénie libre des vaches laitières. Ann. Biol. anim. Bioch. Biophys., 10, 517-521.

Champlemox C., Pion R., Thrend P., ig7r. Composition en acides aminés des matières azotíx du contenu duochinal du ruminant. Xe Congris Internat. Zootech., Versailles.

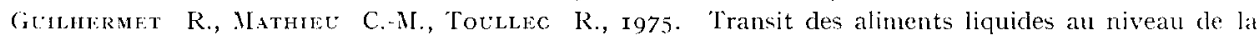
gouttic̀re osophagiente chez le veau préruminant et ruminant. Ann. Zootech., 24, 69-79.

Leibnol\% J., Narion R. W., r97I. The effect of urea in the diet of the early weaned calf on weight gain, nitrogen and sulphur balance, and plasma urea and free amino acid concentration. Aust. J. Agric. Res., 22, 65.5-662.

LeRoY F., ZeLTer S.-Z., I97o. P'rotection des protéines alinentaires contre la désamination bactérienne au niveau du rumen. II. Ëtude in vivo sur moutons fistulisés. Ann. Biol. anim. Bioch. Biophys., 10, $40 I-412$.

Mathieg C.-M., Wegat-Litre İ, ig6r. Mise au point d'une méthode d'alimentation des veaux d'álevage. I. Détermination de la quantité de lait nécessaire. Ann. Zootech., 10, I6 I-I75.

Orskoy E. R., J3Exile D., I 969 . Studies on the oesophageal groove reflex in sheep and on the potential use of the groove to prevent the fermentation of food in the rumen. Br. J. Nutr., 23, 4I5-420.

Orsioy li. R., IRaser C., Corse E. L., I970. The effect on protein utilisation of fecding different protein supplements via the rumen or via the abomasum in young growing sheep. Br. J. Nutr., 24, $803-809$.

Patereal-Miraxid P', Pregnate J., Pion R., ig7r. Influence de la nature des proténes des aliments d'allaitenent sur l'aninuacidémie libre du veau préruminant. I' Congrès Internat. Zootech., Versailles. 
Patureau-Mrrand P., Touldec R., Paruelle J.-L., Prugnaud J., Pion R., Ig74. Influence de la nature des matières azotées des aliments d'allaitement sur l'aminoacidémie du vean préruminant. I. Matières azotées du lait, du lactosérum, du poisson et des levures d'alcanes. Ann. Zootech., 23, 343358 .

Pryor W. J., Ternouth J. H., Ig72. A comparison of feeding reconstitued and dried milk upon the growth and carcass composition of calves. Hr. J. Nutr., 27, 5 17-525.

Raven A. M., Robinson K. L., 1964. Factors affecting the nutritive value of fat for calves. II, The system of feeding and the mode of incorporation into meal mixtures. J. Sci. Fd A gric., 15, 2 I9-227.

Vérité R., Hoden A., Journet M., 1974. Ctilisation des tourteaux traités au formol par les varhes laitic̀res, Bull. Tech., Theix, 18, 5-10.

\section{I.N.R.A \\ BIBLIOTHEQUE UO 35906 \\ DOMAINE DE CROUELLE}

63039

CLERMONT-FD CEBEX 2 